\title{
LA SOLUCIÓN DE ALGUNAS ECUACIONES DIFERENCIALES LINEALES DE SEGUNDO ORDEN
}

Fecha de recepción: 26 de octubre de 2015 • Fecha de Evaluación: 16 de febrero de 2016 •

Fecha de aceptación: 18 de marzo de 2016

\section{THE SOLUTION OF SOME SECOND-ORDER LINEAR DIFFERENTIAL EQUATIONS}

José Alfredo Jiménez Moscoso', Jorge Mauricio Ruiz Vera

\section{RESUMEN}

En este artículo, se presenta un nuevo método rápido, eficiente y preciso para determinar la solución general de la ecuación diferencial lineal de segundo orden cuando los coeficientes son variables que se relacionan entre si mediante otra ecuación diferencial ordinaria. Uno de los métodos de solución consiste en que la ecuación diferencial de segundo orden se transforma de una vez a una ecuación diferencial ordinaria de Riccati, ésta última EDO se puede resolver sin necesidad de conocer a priori una solución particular. Estos métodos de solución brindan herramientas que permiten explicar este tipo de EDO de forma simple en las aulas.

Palabras clave: Ecuación diferencial de segundo orden con coeficientes variables, Ecuación exacta, Ecuaciones diferenciales ordinarias lineales.

1 Departamento de estadísitca. Facultad de Ciencias, Universidad Nacional de Colombia

2 Departamento de matemáticas, Facultad de Ciencias, Universidad Nacional de Colombia *Autor corresponsal. E-mail: jmruizv@unal.edu.co. 


\begin{abstract}
In this paper we present a new fast, efficient and accurate way to determine the general solution of secondorder linear differential equation when the coefficients are variable and are related to each other by another ordinary differential equation. One of the solution methods proposed is that the second-order differential equation is transformed in a Riccati ordinary differential equation, such ODE latter can be solved without knowing a priori a particular solution. These solution methods provide tools that explain this kind of ODE in a simple way in the classroom.
\end{abstract}

keywords: Second-order linear differential equation with variable coefficients, exact equation, linear ordinary differential equations.

\title{
INTRODUCCIÓN
}

Una ecuación diferencial ordinaria (EDO) lineal de segundo orden constituye una clase especial de ecuaciones diferenciales, y juega un papel importante en muchos campos de la ciencia aplicada, este tipo de EDO aparece en problemas clásicos de mecánica, electromagnetismo y dinámica de gases (Murphy 1960; Boyce y DiPrima 1997). Por lo general, una EDO lineal de la forma

$$
a_{2}(x) y^{\prime \prime}(x)+a_{1}(x) y^{\prime}(x)+a_{0}(x) y(x)=f(x),
$$

donde los coeficientes variables $a_{2}(x), a_{1}(x), a_{0}(x)$ y $f(x)$ son funciones arbitrarias de $x$ con $a_{2}(x) \neq 0$, $a_{1}(x) \neq 0$, se denomina EDO lineal de segundo orden y para resolverla se necesita que los coeficientes $a_{k}(x)$ satisfagan ciertas condiciones. La forma más conocida consiste en dividir la EDO (1) entre $a_{2}(x)$ y escribirla en su forma canónica:

$$
y^{\prime \prime}(x)+P(x) y^{\prime}(x)+Q(x) y(x)=g(x) .
$$

El procedimiento para determinar la solución de esta EDO lineal de segundo orden tiene dos pasos: primero se resuelve la EDO lineal homogénea asociada y luego empleando el método de variación de parámetros se determina una solución particular, la solución general de la EDO no homogénea completa se logra sumando estas dos soluciones (veáse Boyce y DiPrima 1997; Nagle et al. 2012; Simmons 1991).

La idea principal del artículo es ofrecer un nuevo enfoque para obtener la solución general de algunas EDO lineales homogéneas de segundo orden, además, mostrar e impulsar el uso de este método de solución entre ingenieros en ejercicio y estudiantes de Ingeniería. Para utilizar el método de solución propuesto se debe satisfacer alguna de las dos condiciones dadas en este trabajo, y cuando se verifica alguna de ellas, la EDO lineal de segundo orden puede reducirse a una ecuación de primer orden, lo que permite solucionarla fácilmente sin utilizar series de potencias.

El artículo está organizado de la siguiente manera: en la sección 1.2 se presentan los métodos de solución existentes; en la sección 1.3 se presentan los métodos de solución propuestos; en la sección 1.4 se presentan algunas aplicaciones de estos métodos, para ello, se seleccionan algunas ecuaciones diferenciales cuyas soluciones se obtienen usando series de 
potencias y se resuelven empleando la metodología propuesta. Finalmente, se presentan las conclusiones.

\section{ANTECEDENTES}

A continuación se describen los métodos de solución de EDO lineales de segundo orden, que se presentan en los textos clásicos de EDO y que empleamos los docentes de matemáticas. Por simplicidad en esta sección se consideran los métodos de solución de EDO lineales homogéneas de segundo orden. Aunque en (Kovacic 1986) se presentan cuatro casos para solucionar de manera cerrada las EDO lineales homogéneas de segundo orden, los cuales se conocen como el Algoritmo de Kovacic, en esta sección no se consideran.

\section{Ecuación de Cauchy-Euler}

Esta EDO lineal es en la que el grado de los coeficientes coincide con el orden $k$ de la diferenciación de la EDO (1), es decir los coeficientes son de la forma:

$$
a_{k}(x)=a_{k} x^{k}, \quad k=0,1,2,
$$

donde $a_{k} \in \mathrm{R}$. Para este tipo de EDO las soluciones son de la forma $y(x)=x^{r}$, y el valor de $r$ se determina como la solución de la ecuación cuadrática:

$$
a_{2} r(r-1)+a_{1} r+a_{0}=0
$$

Este tipo de EDO tiene una de las siguientes soluciones:

1. Si $r_{1} \neq r_{2}$ y son reales, entonces $y(x)=c_{1} x^{r_{1}}+c_{2} x^{r_{2}}$,

2. Si $r_{1}=r_{2}, \quad y(x)=c_{1} x^{r_{1}}+c_{2} x^{r_{2}} \ln x$,

3. $\mathrm{Si}$

$$
r_{1}=a+i b, \quad r_{2}=a-i b,
$$

$y(x)=x^{a}\left(c_{1} \cos (b \ln x)+c_{2} \sin (b \ln x)\right.$.

\section{Ecuación exacta}

Es aquella en la que los coeficientes de la EDO (1) satisfacen el siguiente test de exactitud ${ }^{1}$ :

$$
a_{2}^{\prime \prime}(x)-a_{1}^{\prime}(x)+a_{0}(x)=0 .
$$

Este tipo de EDO tiene una solución de la forma

$$
y_{1}(x)=a_{2}(x) \exp \left\{-\int \frac{a_{1}(x)}{a_{2}(x)} \mathrm{d} x\right\}
$$

y la otra solución se determina usando el método de reducción de orden.

\section{Cambio de variable}

El cambio de función $y(x)$ por $u(x)$ dado por $y(x)=\exp \left\{-\int u(x) \mathrm{d} x\right\}$ transforma la EDO (1) en

$$
u^{\prime}(x)=(u(x))^{2}-\frac{a_{1}(x)}{a_{2}(x)} u(x)+\frac{a_{0}(x)}{a_{2}(x)}
$$

Esta es una ecuación de Riccati. En algunos casos poco se gana mediante esta transformación, ya que la nueva EDO (3) puede ser tan difícil de resolver como la anterior. En (Sugai 1961) se presentan varias transformaciones que permiten linealizar la EDO de Riccati y para algunos casos determinar la solución general de dicha EDO. En (Jiménez 2015) se presentan algunas soluciones de la EDO de Riccati cuando los coeficientes satisfacen ciertas condiciones, lo cual permite encontrar la solución general sin conocer una solución particular.

\section{Solución mediante series de potencias}

Entre los tipos de EDOs lineales que se resuelven por este método se encuentran: la ecuación de Airy, las ecuaciones de Bessel, las ecuaciones de Legendre y la ecuación hipergeométrica, entre otras.

1 En (Murphy 1960) se presenta la generalización de este test para EDO lineales de orden superior. 


\section{MÉTODOS PROPUESTOS}

Los métodos que se proponen para establecer una solución de la EDO lineal homogénea asociada a la EDO dada en (1) con coeficientes variables, se resumen en los teoremas que se presentan a continuación:

Teorema 1. Si los coeficientes de la EDO lineal dada en (1) están relacionados mediante la siguiente condición

$$
\frac{a_{0}(x)}{a_{2}(x)}=\frac{\mathrm{d}}{\mathrm{d} x}\left[\frac{a_{1}(x)}{a_{2}(x)}\right]
$$

entonces una solución de la ecuación homogénea asociada viene dada por

$$
y_{1}(x)=\exp \left\{-\int \frac{a_{1}(x)}{a_{2}(x)} \mathrm{d} x\right\},
$$

y por el método de reducción de orden: $y_{2}(x)=y_{1}(x) u(x)$, donde

$$
u(x)=\int \exp \left\{\int \frac{a_{1}(x)}{a_{2}(x)} \mathrm{d} x\right\} \mathrm{d} x .
$$

Prueba. Si los coeficientes satisfacen la expresión (4), entonces la EDO lineal homogénea asociada a la EDO lineal de segundo orden (1) se puede reescribir como

$$
\begin{gathered}
a_{2}(x)\left[y^{\prime \prime}(x)+\frac{a_{1}(x)}{a_{2}(x)} y^{\prime}(x)+\frac{\mathrm{d}}{\mathrm{d} x}\left(\frac{a_{1}(x)}{a_{2}(x)}\right) y(x)\right]=0 \\
a_{2}(x) \frac{\mathrm{d}}{\mathrm{d} x}\left[y^{\prime}(x)+\frac{a_{1}(x)}{a_{2}(x)} y(x)\right]=0,
\end{gathered}
$$

esta expresión se comporta como la derivada de una constante, luego

$$
\kappa=y^{\prime}(x)+\frac{a_{1}(x)}{a_{2}(x)} y(x),
$$

con $\kappa \in \mathrm{R}$, esta EDO es lineal de primer orden y usando el factor integrante

$$
\mu(x)=\exp \left\{\int \frac{a_{1}(x)}{a_{2}(x)} \mathrm{d} x\right\},
$$

así se llega a la siguiente solución

$$
y(x)=\exp \left\{-\int \frac{a_{1}(x)}{a_{2}(x)} \mathrm{d} x\right\}\left[\int \kappa \exp \left\{\int \frac{a_{1}(x)}{a_{2}(x)} \mathrm{d} x\right\} \mathrm{d} x+E\right],
$$

donde $E \in \mathrm{R}$ y se tiene lo que se quería demostrar.

Teorema 2. Si los coeficientes de la EDO lineal dada en (1) satisfacen la siguiente condición

$$
\frac{\mathrm{d}}{\mathrm{d} x}\left[\frac{a_{1}(x)}{a_{0}(x)}\right]=-1,
$$

entonces una solución de la ecuación homogénea asociada es

$$
y_{1}(x)=\frac{a_{1}(x)}{a_{0}(x)},
$$

y por el método de reducción de orden:

$$
\begin{aligned}
& y_{2}(x)=\frac{a_{1}(x)}{a_{0}(x)} u(x) \text {, donde } \\
& u(x)=\int \exp \left\{\int\left[2 \frac{a_{0}(x)}{a_{1}(x)}-\frac{a_{1}(x)}{a_{2}(x)}\right] \mathrm{d} x\right\} \mathrm{d} x .
\end{aligned}
$$

Prueba. Si los coeficientes satisfacen la expresión (5), entonces es sencillo verificar que una solución de la EDO lineal homogénea asociada a la EDO lineal de segundo orden (1) viene dada por

$$
y_{1}(x)=\frac{a_{1}(x)}{a_{0}(x)} .
$$

Empleando el método de reducción de orden se tiene

$$
\begin{gathered}
y_{2}(x)=y_{1}(x) u(x), \quad y_{2}^{\prime}(x)=y_{1}(x) u^{\prime}(x)-u(x), \\
y_{2}^{\prime \prime}(x)=y_{1}(x) u^{\prime \prime}(x)-2 u^{\prime}(x),
\end{gathered}
$$

la EDO lineal dada en (1) se convierte en

$$
a_{2}(x)\left[y_{1}(x) u^{\prime \prime}(x)-2 u^{\prime}(x)\right]+a_{1}(x) y_{1}(x) u^{\prime}(x)=0,
$$

luego, se llega a la siguiente solución

$u(x)=\int \exp \left\{\int\left[\frac{2}{y_{1}(x)}-\frac{a_{1}(x)}{a_{2}(x)}\right] \mathrm{d} x\right\} \mathrm{d} x=\int \frac{\exp \left\{-\int \frac{a_{1}(x)}{a_{2}(x)} \mathrm{d} x\right\}}{\left(y_{1}(x)\right)^{2}} \mathrm{~d} x$, sustituyendo (6) se tiene lo que se quería demostrar. 
En otras palabras, el Teorema 2 nos dice que en caso que el cociente entre $a_{1}(x)$ y $a_{2}(x)$ sea una función lineal con pendiente de valor -1 una solución de la EDO lineal homogénea (1), es dicha función lineal.

\section{ILUSTRACIÓN}

En esta sección, se aplican los métodos presentados para encontrar la solución general de algunas EDO lineales de segundo orden dadas en (Boyce y DiPrima 1997; Spiegel 1983; Simmons 1991).

Ejemplo 1. Encuentre la solución general de la EDO lineal homogénea

$$
2 x^{2} y^{\prime \prime}+x(2 x+1) y^{\prime}-y=0, \quad x>0 .
$$

Solución. Este ejercicio es uno de los ejemplos que aparece en (Simmons 1991, página 195) para ilustrar el método de Frobenius. Nótese que los coeficientes de esta EDO satisfacen la condición dada en (4), luego, la EDO lineal se puede reescribir como

$$
2 x^{2} \frac{\mathrm{d}}{\mathrm{d} x}\left(y^{\prime}+\frac{2 x+1}{2 x} y\right)=0 \text {, }
$$

luego

$$
y^{\prime}+\frac{2 x+1}{2 x} y=k,
$$

mediante el uso del factor integrante, la solución general es:

$$
y(x)=\frac{1}{\sqrt{x}} e^{-x}\left[c_{1}+k \int \sqrt{x} e^{x} \mathrm{~d} x\right]=c_{1} y_{1}(x)+c_{2} y_{2}(x) .
$$

donde $y_{1}(x)$ corresponde a la solución de la EDO lineal de primer orden que aparece en la expresión (7). Nótese que esta EDO lineal no es exacta. Además, el procedimiento propuesto involucra pocos pasos a diferencia del método de solución en series.

Ejemplo 2. Encuentre la solución general de la EDO lineal homogénea

$$
y^{\prime \prime}+x y^{\prime}+y=0
$$

Solución. Este ejercicio aparece propuesto en (Boyce y DiPrima 1997, página 139) para aplicar el método de exactas. Nótese que los coeficientes de esta EDO satisfacen la condición dada en (4), luego, la EDO lineal se puede reescribir como

$$
\frac{\mathrm{d}}{\mathrm{d} x}\left(y^{\prime}+x y\right)=0,
$$

esta nueva EDO se puede reescribir como

$$
\frac{\mathrm{d} y}{\mathrm{~d} x}+x y=k
$$

la cual corresponde a una EDO lineal de primer orden y su solución viene dada por

$$
y=e^{-\frac{1}{2} x^{2}}\left[\int k e^{\frac{1}{2} x^{2}} \mathrm{~d} x+C\right] .
$$

Por lo tanto, la solución general es:

$$
y(x)=c_{1} e^{-\frac{1}{2} x^{2}}+c_{2} e^{-\frac{1}{2} x^{2}} \int e^{\frac{1}{2} x^{2}} \mathrm{~d} x
$$

nótese que esta EDO lineal es exacta. Se puede apreciar que el procedimiento es mucho más directo y sencillo que el método de solución de EDO exactas.

Ejemplo 3. Encuentre la solución de la EDO lineal de Legendre

$$
\left(1-x^{2}\right) y^{\prime \prime}-2 x y^{\prime}+2 y=0 .
$$

Solución. Este ejercicio aparece propuesto en (Spiegel 1983, página 351) para solucionarlo usando polinomios de Legendre. Nótese que los coeficientes de esta EDO satisfacen la condición dada en (5), luego, una solución de esta EDO de segundo orden es

$$
y_{1}(x)=x,
$$

y la otra solución viene dada por 


$$
\begin{gathered}
y_{2}(x)=x \int \exp \left\{\int\left[-\frac{2}{x}+\frac{2 x}{1-x^{2}}\right] \mathrm{d} x\right\} \mathrm{d} x \\
=x \int \exp \left\{\int\left[-\frac{2}{x}+\frac{1}{1-x}-\frac{1}{1+x}\right] \mathrm{d} x\right\} \mathrm{d} x \\
=x \int \frac{\mathrm{d} x}{x^{2}\left(1-x^{2}\right)},
\end{gathered}
$$

nuevamente usando fracciones parciales, después de algunas operaciones y simplificando se llega a

$$
y_{2}(x)=x \tanh ^{-1}(x)-1,
$$

donde $\tanh ^{-1}(x)=\operatorname{arctanh}(x)$. Por lo tanto, la solución general es:

$$
y(x)=c_{1} x+c_{2}\left(x \tanh ^{-1}(x)-1\right) .
$$

Nótese que esta EDO lineal no es exacta. Además, el procedimiento propuesto involucra pocos pasos a diferencia del método de solución en series.

A continuación se presentan dos ejemplos prácticos, donde los métodos propuestos en este artículo pueden aplicarse.

\section{Ejemplo 4. Resistencia eléctrica variable}

Considere un circuito resistor-inductor-condensador $R L C$ mostrado en la figura. Suponga que el voltaje en la fuente es $E(t)=0$ voltios, la inductancia es $L$ henrios, la capacitancia es $C$ faradios. Además, se supone que el resistor se calienta, tal que la resistencia eléctrica aumenta de la forma $R(t)=R_{0}+\frac{1}{C} t$ ohmios, donde $R_{0}$ es una constante que denota la resistencia de referencia del resistor (Halliday et al. 1999). Determine la carga del condensador en el circuito.

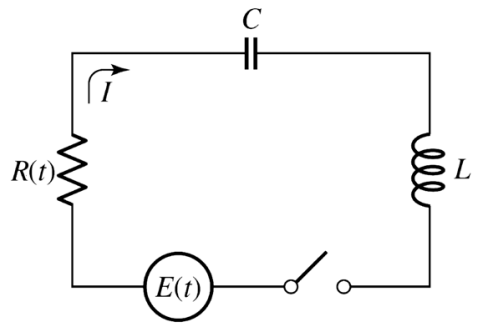

Figura 1: Circuito RLC con resistencia variable.

Solución. La carga $q(t)$ del condensador es la solución de la ecuación de segundo orden

(8)

$$
L \frac{d^{2}}{d t^{2}} q(t)+\left(R_{0}+\frac{1}{C} t\right) \frac{d}{d t} q(t)+\frac{1}{C} q(t)=0 .
$$

Obsérvese que la condición (4) la satisface la ecuación, por lo cual la EDO lineal de segundo orden puede escribirse como

$$
L \frac{d}{d t}\left\{\frac{d}{d t} q(t)+\frac{1}{L}\left(R_{0}+\frac{1}{C} t\right) q(t)\right\}=0 .
$$

Para encontrar una solución al problema homogéneo (8) se resuelve la ecuación lineal exacta

$$
\frac{d}{d t} q(t)+\frac{1}{L}\left(R_{0}+\frac{1}{C} t\right) q(t)=k_{1}, \quad\left(\mathrm{k}_{1} \text { constante }\right)
$$

cuya solución general es

$$
q(t)=\exp \left\{-\frac{R_{0}}{L} t-\frac{1}{2 L C} t^{2}\right\}\left[k_{2}+k_{1} \int \exp \left\{\frac{R_{0}}{L} t+\frac{1}{2 L C} t^{2}\right\} d t\right],
$$

donde $k_{2}$ es constante.

Ejemplo 4. Dinámica en un ecosistema marino

Denotando por $x$ y $y$ las cantidades de biomasa de zooplancton y fitoplancton en cierto ecosistema 
marino respectivamente. Suponiendo la gran abundancia de fitoplancton en el ecosistema y dado que éste constituye el alimento del zooplancton (Pastor 2008), entonces se considera que la razón de cambio de biomasa de zooplancton es proporcional a la cantidad de biomasa de fitoplancton. Además se supone que el fitoplancton crece con una tasa intrinseca variable en el tiempo de la forma $1+e^{-t}$ y la biomasa de fitoplancton decrece debido a los organismos consumidores (zooplancton) con una razón variable en el tiempo de la forma $e^{-t}$. Entonces la interacción entre las dos poblaciones de organismos, se modela por el siguiente sistema de ecuaciones diferenciales

$$
\begin{aligned}
& \frac{d x}{d t}=y \\
& \frac{d y}{d t}=\left(1+e^{-t}\right) y-\mathrm{e}^{-t} x
\end{aligned}
$$

Determine la cantidad de biomasa de ambas poblaciones.

Solución. Primero se transforma el sistema de ecuaciones diferenciales de primer orden (9) en una ecuación diferencial de segundo orden. Derivando una vez la primera ecuación de (9) y sustituyendo $\frac{d y}{d t}$, se obtiene.

$$
\frac{d^{2} x}{d t^{2}}-\left(1+e^{-t}\right) \frac{d x}{d t}+\mathrm{e}^{-t} x=0
$$

Obsérvese que la condición (4) la satisface la ecuación (10), por lo cual se puede escribir como

$$
\frac{d}{d t}\left(\frac{d x}{d t}-\left(1+e^{-t}\right) x\right)=0
$$

Ahora se determina una solución al problema homogéneo (10) resolviendo la ecuación lineal exacta

$$
\frac{d x}{d t}-\left(1+e^{-t}\right) x=k_{1}, \quad\left(k_{1} \text { constante }\right)
$$

La solución general es entonces

$$
x(t)=\exp \left\{t-e^{-t}\right\}\left[k_{2}+k_{1} \int \exp \left\{e^{-t}-t\right\} d t\right],
$$

donde $k_{2}$ es constante. Finalmente de la ecuación (11) se obtiene que la biomasa del fitoplancton es

$$
y(t)=\left(1+e^{-t}\right) x(t)+k_{1}
$$

\section{CONCLUSIONES}

En este artículo se presenta un nuevo método de solución de algunas EDO lineales de segundo orden con coeficientes variables. Cuando los coeficientes de la EDO satisfacen alguna de las condiciones establecidas, el método permite determinar de manera exacta y muy sencilla la solución general de la EDO sin necesidad de emplear series de potencias. Las condiciones propuestas sobre los coeficientes de la $E D O$, se pueden ver como un criterio para reducir la ecuación a primer orden. En trabajos futuros se desea identificar criterios de este estilo para EDO de orden superior. 


\section{REFERENCIAS}

1. Boyce $W$ y R DiPrima 1997. Elementary Differential Equations and Boundary Value Problems. Sexta edición, John Wiley \& Sons, New York, 749p.

2. Halliday D, R Resnick y K Krane. 1999. Física. Volumen 2, Cuarta edición. Compañía Editorial Continental, S.A. De C.V, México, 758p.

3. Jiménez J. A. 2015. La solución de algunas EDO de Riccati. Revista digital, Matemática, Educación e Internet. 15(2):1-14.

4. Kovacic J. J. 1986. An Algorithm for Solving Second Order Linear Homogeneous Differential Equations. Journal of Symbolic Computation. 2:3-43.

5. Murphy G 1960. Ordinary differential equations and their solutions. Van Nostrand Reinhold Company, New York, 462p.

6. Nagle R, E Saff y A Snider 2012. Fundamentals of Differential Equations and Boundary Value Problems. Sexta edición, Addison-Wesley, New York, 816p.
7. Pastor J 2008. Mathematical Ecology of Populations and Ecosystems. Wiley-Blackwell, Oxford, 344p.

8. Simmons G 1991. Differential Equations with Applications and Historical Notes. Segunda edición, McGraw-Hill, New York, 629p.

9. Spiegel M 1983. Ecuaciones diferenciales aplicadas. Tercera edición, Prentice-Hall Hispanoamericana, S.A. México, 662p.

10. Sugai I. 1961. A class of solved Riccati's equations. Electrical Communication, 37(1):56-60.

11. Zwillinger D 1997. Handbook of Differential Equations. Tercera edición, Academic Press, Boston, 787p. 